\title{
A Study on Prevalence of Left Ventricular Dysfunction And its Correlation With Estimated Glomerular Filtration Rate (Egfr) In Ckd Patients
}

\author{
Chelliah Dharmaraj ${ }^{1}$,Tina Ann Antony ${ }^{2}$, Gokul Prasannan ${ }^{3}$ \\ ${ }^{I}$ Professor, Department Of General Medicine, Madurai Medical College \\ ${ }^{2}$ Assistant Professor, Department Of General Medicine,Sree Narayana Institute Of Medical \\ Sciences,Ernakulam \\ ${ }^{3}$ Post Graduate,Department Of General Medicine,Madurai Medical College
}

\begin{abstract}
Context: Chronic kidney disease (CKD) is a spectrum of pathophysiological process that is associated with dysfunction of kidney and the progressive decrease in Glomerular Filtration Rate $(G F R) .{ }^{(1)}$ Strategies to prevent the development and progression of $L V$ dysfunction at an early stage may prove more effective.

Aim Of The Study: To assess the presence of Left Ventricular (systolic and/ or diastolic) dysfunction in patients with CKD. To evaluate the correlation between Left Ventricular dysfunction and eGFR in CKD patients To compare the Left Ventricular dysfunction in hypertensive and normotensive CKD patients

Settings And Design: Cross sectional analytical study

Materials And Methods: This study is to be conducted among 100 established CKD patients from May 2014 to August 2014,who are admitted in Govt. Rajaji Hospital, Madurai in the General Medicine and Nephrology Departments and 50 age and sex matched controls. Out of the 100

CKD patients, 50 will be hypertensive and 50 will be normotensive CKD patients.Cases were classified into different stages of Chronic Kidney Disease based on estimated Glomerular filtration rate (eGFR). eGFR was calculated using MDRD formula.

Statistical Analysis: Mean,chi-square test,pearson'"s coefficient

Results: There is significant association between CKD and LV diastolic dysfunction.There is a moderate negative correlation between eGFRand diastolic dysfunction. That is as the eGFRdecreases, the diastolic dysfunction increases.

Conclusions: Diastolic dysfunction is first to appear in CKD patients and will be present in more number of patients as compared to systolic dysfunction.Lower the eGFR more severe will be the LV dysfunction.CKD patients with hypertension and anemia will have more severe LV dysfunction.
\end{abstract}

Keywords: Chronic kidney disease,estimated GFR, left ventricular dysfunction.

\section{Introduction}

Chronic kidney disease (CKD) is a spectrum of pathophysiological process that is associated with dysfunction of kidney and the progressive decreasein Glomerular Filtration Rate (GFR). It is a functional or structural abnormalities of kidneys for more than three months, irrespective of cause. It is a global health concern. CKD has been the $12^{\text {th }}$ leading cause of death, and the $17^{\text {th }}$ leading cause of disability. CKD has a number of co-morbidities and hence a disease with high mortality. An analysis of risk factors for development and progression of CKD is necessary in clinical practice. In Chronic Kidney Disease there is a higher incidence of cardiovascular events. Cardiovascular cause is one of the prime causes of morbidity and mortality in all the stage of CKD. Most of the patients with CKD develop cardiovascular disease before reaching ESRD. The CKD registry $^{(6)}$ has shown patients with CKD stage 4 have a high incidence of cardiovascular complications. Most of the patients die before they have ESRD. Patients in early stages of CKD are also found to have a high cardiovascular morbidity. Hence we should look into the prevention of cardiovascular complications.

A large cohort study of patients with CKD stages 2 to 4 showed that death was seen more often than progression to kidney failure in all stages of CKD. There was a high baseline prevalence of cardiovascular disease in the patients who died, compared to those who survived, which suggests that cardiovascular disease accounted for a huge proportion of the deaths. Hence, most patients in the earlier stages of CKD do not progress to kidney failure because ofthe mortality caused due to cardiovascular disease. Cardiovascular disease has been seen as a major "competing outcome or risk" of kidney failure. ${ }^{(3)}$ 


\section{The Cardiovascular Manifestation In CKD Includes:}

- Ischemic heart disease - myocardial infarction and angina

- Left ventricular hypertrophy

- Heart failure : LV diastolic and systolic dysfunction and

- Dilated cardiomyopathy.

Heart and kidney are linked to each other in the hemodynamic and regulatory functions. There are multiple communication among the two organs like Sympathetic Nervous System, Anti Diuretic Hormone, Renin Angiotensin Aldosterone System and natriuretic peptides. ${ }^{(8)}$ National Kidney Foundation formed a Task Force which considered Coronary Artery Disease (CAD) and LVH which are the target conditions, ecommended for decreasing cardiovascular mortality in ESRD. LVH is associated with both systolic and diastolic dysfunction. LVH associated with systolic dysfunction is expressed by decreased mid wall systolic fractional shortening and decreased ejection fraction. These findings shows us that mild reduction in the renal perfusion which is caused by the slightly impaired LV systolic dysfunction, which is associated with pathological, highly pulsatile perfusion in the microvasculature of the kidney might be mechanisms through which a progressive reduction of renal function takes place in patients with pre-existing renal damage. This renal insufficiency produced, adversely affectsfunction of the heart, which produces a vicious cycle were the renal failure further impairs cardiac performance.

Diastolic dysfunction is associated with higher incidence of intradialytic hypotension and higher perioperative death from pulmonary oedema at the time of renal transplantation. It is significant to recognize and correct the cardiovascular complications and risk factors before the commencement of dialysis.

Echocardiography will provide us with a very simple and non-invasive assessment of the structure and function of the heart. It also helps us to identify the people who are at greater risk. Strategies to prevent the development and progression of $\mathrm{LV}$ dysfunction at an early stage may prove more effective.

\section{Materials And Methods}

This study is to be conducted among 100 established CKD patients who are admitted in Govt. Rajaji Hospital, Madurai in the General Medicine and Nephrology Departments and 50 age and sex matched controls. Out of the 100 CKD patients, 50 will be hypertensive and 50 will benormotensive CKD patients. Cases were classified into different stages of Chronic Kidney Disease based on estimated Glomerular filtration rate (eGFR). eGFR was calculated using MDRD formula.

\section{Inclusion Criteria:}

Age > 18 years, both sex, with established Chronic Kidney Diseasei.e.: Symptoms and signs of renal disease > 3 months

Proteinuria $>1 \mathrm{~g} / \mathrm{dl}$

Ultrasonography showing kidney size $<8.5 \mathrm{~cm}$

Reduced creatinine clearance (eGFR) pointing to CKD

Those who have not received any forms of renal

replacement therapy.

50 hypertensive (BP > 140/90) and 50 normotensive CKD patients in whom the duration of CKD is matched.

0 sex and age matched healthy individuals as controls.

\section{Exclusion Criteria:}

Age $<18$ years and $>70$ years Patients with Acute Kidney Injury Patients with history and clinical features suggestive of pre-existing cardiac diseases like rheumatic valvular heart disease, congenital heart disease, coronary heart disease, cardiomyopathy and pericardial diseases. Patients on dialysis and kidney transplant patients

\section{Ethical Committee Approval: Obtained.}

Study Protocol:

A previously designed proforma will be used to collect the demographic and clinical details of the patients and controls. A thorough clinical examination will be done. An ECG and an Ultrasound Abdomen will be taken. Blood investigations taken. Echocardiography will be taken according to the standard guidelines. All measurements were made following American Society of Echocardiography (ASE) recommendations Blood samples were collected from all participants to estimate Haemoglobin. Blood urea, and Serum creatinine. Urine sample will be analysed for albuminuria. 


\section{Statistical Analysis}

The final data was entered onto Microsoft excel sheet 2010 and statistical analysis was done using SPSS software. The statistical tools applied were median, mean,standard deviation, Pearson's correlation and chi - square testcoefficient. These results are considered very significant when the $\mathrm{p}$ value $<0.01$ and significant if $\mathrm{p}$ value $<0.05$

\section{Results}

About $52 \%$ of study subjects were in the age group of $41-60$ years while $36 \%$ were aged $61-70$ years Majority of the study subjects were males (70\%) while the remaining $30 \%$ were females. $84 \%$ of CKD with hypertension had diastolic dysfunction, while only $26 \%$ of the same group had systolic dysfunction. $74 \%$ of CKD patients have diastolic dysfunction as compared to $16 \%$ in controls. This association was found to be extremely significant by Chi square test. $64 \%$ of CKD with normotension have diastolic dysfunction as compared to $16 \%$ in controls. This association was found to be statistically significant There is a higher prevalence of anaemia as CKD progresses, $\mathrm{Hb}$ was found to be $>7 \mathrm{gm} / \mathrm{dl}$ in all patients in stage $3 \mathrm{CKD}$ and < $7 \mathrm{gm} / \mathrm{dl}$ in $28 \%$ patients in stage 5 . According to Pearson's correlation coefficient; the correlation between eGFR and diastolic dysfunction was calculated and $\mathrm{R}$ value obtained was $\mathrm{R}=-0.90682$. This showed that there is a moderate negative correlation betweeneGFRand diastolic dysfunction. That is as the eGFRdecreases, the diastolic dysfunction increases.

Prevalence Of Lv Diastolic Dysfunction In Ckd

\begin{tabular}{|l|l|l|l|}
\hline & $\begin{array}{l}\text { DD } \\
\text { PRESENT }\end{array}$ & DD ABSENT & TOTAL \\
\hline CKD & 74 & 26 & 100 \\
\hline CONTROLS & 8 & 42 & 50 \\
\hline TOTAL & 82 & 68 & 150 \\
\hline
\end{tabular}

Comparison Of Diastolic Dysfunction In Ckd + Htn And Ckd + Nt

\begin{tabular}{|l|l|l|l|}
\hline & DD PRESENT & DD ABSENT & TOTAL \\
\hline CKD + HTN & 42 & 8 & 50 \\
\hline CKD + NT & 32 & 18 & 50 \\
\hline TOTAL & 64 & 26 & 100 \\
\hline
\end{tabular}

Chi square $\mathrm{p}$ value $=0.022$

Prevalence Of Anaemia In Study Group

\begin{tabular}{|l|l|l|l|}
\hline & $<\mathbf{7}$ & $\mathbf{7 - 1 0}$ & $>\mathbf{1 0}$ \\
\hline STAGE 3 & 0 & 17 & 2 \\
\hline STAGE 4 & 5 & 24 & 1 \\
\hline STAGE 5 & 28 & 22 & 1 \\
\hline CONTROLS & 0 & 0 & 0 \\
\hline
\end{tabular}

\section{Discussion}

Various epidemiological studies shows that a moderate reduction in kidney function is associated with increased cardiovascular risk, and that the level of kidney function is an independent predictor of cardiovascular outcome The main aim of the study was to demonstrate the prevalence of left ventricular dysfunction in CKD as compared to that of controls and its correlation with the eGFR. The association of various parameters like sex, age, BMI, LVH was also observed. In this study, the study participants were divided into three groups. 50 hypertensive CKD patients, 50 normotensive CKD patients and 50 controls being age and sex matched. It was observed that there was a declining trend in eGFR and $\mathrm{Hb}$ with the progression of renal failure, while there was an ascending trend with systolic and diastolic blood pressure and S.creatinine.

CKD hypertensives have a very low eGFR as compared to that of controls.

\section{Left ventricular Hypertrophy:}

In my study $80 \%$ of CKD hypertensives had LVH as compared to $64 \%$ normotensives. Also $55 \%$ of population who had LVH were CKD hypertensives. This is in accordance with the study done by Levin et al which showed that $70 \%$ of ESRD patients had LVH ${ }^{(9)}$ anPaoletti et al 74\% had LVH

\section{LV systolic dysfunction:}

$26 \%$ of CKD with hypertensives and $8 \%$ of CKD normotensives had systolic dysfunction. This was found to be statistically significant, but when CKD normotensives were compared with that of controls, this association was found to be not significant.This is similar to the study conducted by Shah Harsh D et al, Raj et al and P. Dangri et al. This suggests that LVEF is well maintained till late. This shows that when the ventricle is 
stressed by a haemodynamic overload, it first uses its compensatory mechanism to maintain normal perfrormance. Its only when all these compensatory mechanisms has been maximally used there is a decline in ejection phase indices.

\section{LV diastolic dysfunction:}

$74 \%$ of CKD patients have diastolic dysfunction as compared to $16 \%$ in controls. This association was found to be extremely significant by Chi square test. The average E/A ratio in CKD hypertensives were $1.75 \pm$ 0.75 . According to Virtenen et al, in Grade 5 CKD, the mean E/A ratio is $1.5 \pm 0.5$. ${ }^{(8)}$. In my study, $84 \%$ of CKD with hypertension have diastolic dysfunction as compared to $64 \%$ in CKD normontensives. This association was found to be statistically significant. The average E/A ratio in CKD normotensives were $1.57 \pm$ 0.89. 64\% of CKD with normotension have diastolic dysfunction as compared to $16 \%$ in controls. This association was found to be statistically significant. $64 \%$ of CKD with normotension have diastolic dysfunction as compared to $16 \%$ in controls. This association was found to be statistically significant. It was found that during the study, among the control group 8 out of 50 has E/A ratio less than 1.These were those who were above 60 years and here diastolic dysfunction was considered physiological. This study shows that there is a statistically significant occurrence of diastolic dysfunction in CKD with normotensives as compared to that of control. This proves that it is not the hemodynamic risk factors such as hypertension and anaemia alone that contributes to LVH and LV dysfunction. The uraemia related risk factors also plays a role. So management should be aimed at not only hypertension and anaemia but also control of uraemia related risk factors. Hypertension,anaemiaand alteration in fluid and electrolyte balance are identified as major determinants of LVH in CKD. However beyond hemodynamic factors, inappropriate activation of RAAS, inflammation, oxidative stress, collagen and muscle growth plays a role. Impairment of diastolic dysfunction can occur early, even in the absence of LVH. So early detection of LV dysfunction in CKD could lead to an improvement in CV outcomes in $\mathrm{CKD}$.

This increased myocardial fibrosis has a central role in diastolic dysfunction. There is also a role for inflammation in diastolic dysfunction as demonstrated by increased CRP. LV diastolic dysfunction is frequent among CKD patients and may produce heart failure and mortality. It has been reported that in ESRD, diastolic dysfunction deteriorates in parallel with LVH. ${ }^{(11,12)}$ The negative outcome is stronger in patients with diastolic failure. ${ }^{(13)}$ Diastolic dysfunction in CKD may occur early even in the absence of LVH According to Pearson's correlation coefficient; the correlation between eGFR and diastolic dysfunction was calculated and $\mathrm{R}$ value obtained was $\mathrm{R}=-0.90682$

This showed that there is a moderate negative correlation between eGFR and diastolic dysfunction. That is as the eGFR decreases, the diastolic dysfunction increases. To conclude, patients with CKD have higher prevalence of diastolic and systolic dysfunction and the diastolic dysfunction antedates the systolic dysfunction. ${ }^{(15,16,17)}$

\section{Conclusion}

Left ventricular diastolicdysfunction is more significant than systolic dysfunction in CKD patients. This is found to occur at an earlier stage of CKD , even without LVH. Thus diastolic dysfunction is a much better predictor for cardiovascular mortality. The lower the eGFR, the more severe the LV diastolic dysfunction. LV diastolic dysfunction is more, if there is associated hypertension and anaemia. So, early identification and treatment of above conditions can retard the cardiovascular morbidity and mortality. Even in the absence of hypertension, LV diastolic dysfunction can occur in CKD. This emphasises on the need for correction of non-hemodynamic factors like secondary hyperparathyroidism, inflammation, oxidative stress, myocardial fibrosis, and altered mineral metabolism.Echocardiography provides a simple, non-invasive investigation that can identify even asymptomatic patients at an earlier stage of CKD. So with this study I would recommend earlier screening of patients for LV diastolic dysfunction.

\section{Acknowledgements}

We express our sincere thanks and gratitude to the Dean, Government Rajaji Hospital and Madurai Medical College for permitting us to conduct this study. We express our deep sense of gratitude to HOD of nephrology,cardiology,biochemistry for the support in the study. We are extremely grateful to all our Assistant Professors and PG Residents of Department of Medicine for their constant source of cheer and encouragement throughout the study. We thank all our patients who have formed the backbone of my study, without them this work would not have been possible. We are also thankful to paramedical staff of all departments for their concern.

There is no financial interest in this study. 


\section{References}

[1]. Michael Chonchol and David. M. Spiegel. The patient with Chronic Kidney Disease :Manual of Nephrology: $-7^{\text {th }}$ edition pg 185 $-192$

[2]. Coresh J, Selvin E, Stevens LA, et al. Prevalence of chronic kidney disease in the United States. JAMA 2007;298:2038-2047

[3]. Cockcroft DW, Gault MH. Prediction of creatinine clearance from serum creatinine. Nephron 1976;16:31-

[4]. Shulman NB, Ford CE, Hall WD, et al. Prognostic value ofserum creatinine and effect of treatment of hypertension onrenal function: results from the Hypertension Detection andFollow-up Program. The Hypertension Detection and FollowupProgram Cooperative Group. Hypertension. 1989;13(Suppl):I80-93.

[5]. Manjunath G, Tighiouart H, Ibrahim H, et al. Level of kidney function as a risk factor for atherosclerotic cardiovascular outcomesin the community. J Am CollCardiol. 2003;41:47-55.

[6]. Manjunath G, Tighiouart H, Coresh J, et al. Level of kidneyfunction as a risk factor for cardiovascular outcomes in theelderly. Kidney Int. 2003;63:1121-1129.

[7]. Henry RM, Kostense PJ, Bos G, et al. Mild renal insufficiencyis associated with increased cardiovascular mortality: TheHoorn Study. Kidney Int.

[8]. London GM, MarchaisSJ et al, Cardiac hypertrophy and arterial alteration in ESRD: hemodynamic factors. Kidney Int 1993; 41(Suppl): S 42- S 49

[9]. organ Peterson GE, de Backer T, Gabriel A, et al. Prevalence andcorrelates of left ventricular hypertrophy in the African AmericanStudy of Kidney Disease Cohort Study. Hypertension.2007;50:1033-1039.

[10]. Cottone S, Mulè G, Guarneri M, et al. Endothelin-1 and F2-isoprostane relate to and predict renal dysfunction in hypertensivpatients. Nephrol Dial Transplant. 2009;24:497-503.

[11]. ate London GM. Left ventricular alteration and end-stage renaldisease. Nephrol Dial Transplant. 2002;17:29-36.

[12]. Fathi R, Isbel N, Haluska B, Case C, Johnson DW, MarwickTH. Correlates of subclinical left ventricular dysfunction in ESRD. Am J Kidney Dis. 2003;41:1016-1025.

[13]. Ahmed A, Rich M, Sanders P, et al. Chronic kidney diseaseassociated mortality in diastolic versus systolicheart failure: a propensity matched study. Am J Cardiol.2007;99:393-398.

[14]. Tsioufis C, Vezali E, Tsiachris D, et al. Left ventricular hypertrophyversus chronic kidney disease as predictors of cardiovascularevents in hypertension: a Greek 6-year-follow-up study. J Hypertens. 2009;27:744-752.

[15]. J., 3 Giovanni Cerasola , Emilio Nardi , Alessandro Palermo et al, Epidemiology and pathophysiology of left ventricular abnormalities in chronic kidney disease: a review JNEPHROL 2011; 24(01): 1- 10.

[16]. RathodNitin R, Ghodasara Malay K , Shah Harsh D Assessment of cardiac dysfunction by 2D echocardiography in patients of chronic kidney disease. JPBMS, 2012, 17 (07)

[17]. E. B. Arodiwe, I. I. Ulasi, C. K. Ijoma ,et al Left Ventricular Diastolic Function in a Predialysis Patient Population. West African Journal of Medicine 2010; 29(4): 225-22. 